\title{
Modeling of Turbulent Patches Statistical Distribution in the Stratified Ocean Layers
}

\author{
A. M. Chukharev ${ }^{1, *}$, K. V. Runovsky ${ }^{2}$, O. E. Kulsha ${ }^{2}$ \\ ${ }^{1}$ Marine Hydrophysical Institute, Russian Academy of Sciences, Sevastopol, Russian Federation \\ ${ }^{2}$ Branch of the M.V. Lomonosov Moscow State University in Sevastopol, Russian Federation \\ *e-mail: alexchukh@mail.ru
}

\begin{abstract}
Model for the spectrum of density pulsations in a stratified layer of fluid is proposed. It assumes presence of the mechanism vertical turbulent exchange resulting from breaking of the internal waves and formation of the turbulent patches. The mechanism is considered to be quite widespread in many regions of the World Ocean. Modeling implies consideration of two sources of fluctuations: internal waves existing in the whole layer and turbulence concentrated within a certain number of patches distributed within the layer under consideration. The scale ranges of the internal waves and turbulence are partially overlapped; at that the maximum scale of turbulent pulsations is limited by a patch size. Basing on the theory of locally isotropic turbulence and assuming that the oscillations inside a patch are described by harmonic functions, it is shown that their local frequency and local amplitude are connected by the analytical relationship. In the model functions, both the amplitude and the phase of oscillations are randomized, white noise is added to them. The major features of influence of various specified characteristics of the patches and internal waves upon the spectrum shape are determined. The experimental data have being analyzed by means of the proposed model permit to evaluate the scales and amount of the patches, as well as their turbulence energy level. The model also exhibits validity of the earlier developed energy approach for defining the scales of turbulent patches.
\end{abstract}

Keywords: stratified layer, vertical exchange, internal waves, microstructure, spectral model, turbulent patch.

DOI: 10.22449/1573-160X-2017-5-31-41

(C) 2017, A. M. Chukharev1,*, K. V. Runovsky², O. E. Kulsha²

(C) 2017, Physical Oceanography

\section{Introduction}

Mixing in the ocean stratified layers has a pronounced feature that consists in density increase with depth leading to a decrease in the intensity of vertical movements. Therefore, until the middle of the $20^{\text {th }}$ century it was believed that there was practically no vertical exchange in the ocean thickness with stable stratification. This fact was reflected in proposals to arrange the radioactive waste disposal at the sea bottom, in particular the Black Sea. But measurements applying high-resolution CTD probes have shown that changes in hydrological characterristics with depth possess a complex intermittent nature, i. e. layers with a certain density gradient alternate with completely intermixed fluid layers which is caused by the action of various physical mechanisms, the most important of which is turbulence.

According to the contemporary conception, a special role in vertical mixing in the stratified layers belongs to internal waves. Steady stratification, obstructing the turbulent exchange, promotes the intense internal wave development. For this reason, the internal waves are the most probable source of turbulence and the formation of the hydrophysical field microstructure in the ocean [1]. Mechanism of local instability and breaking of quasi-horizontal shear flows created by inertialgravitational internal waves is considered to make the main contribution to vertical 
turbulent exchange in many World Ocean regions [2]. Numerous observations in the ocean confirm the fact that turbulence in stratified flows is generated by nonregular instability disturbance events leading to the appearance of separate turbulent patches [3]. Turbulent mixing inside such patches results in the formation of vertical heat and mass fluxes in natural currents. The spectra constructed on the basis of the pulsating value measurements in the probing process in such layers have some common features which were described in [4]. Fig. 1 shows the scheme of the temperature gradient pulsation spectrum, where the sections corresponding to internal waves and turbulence can be seen [4].

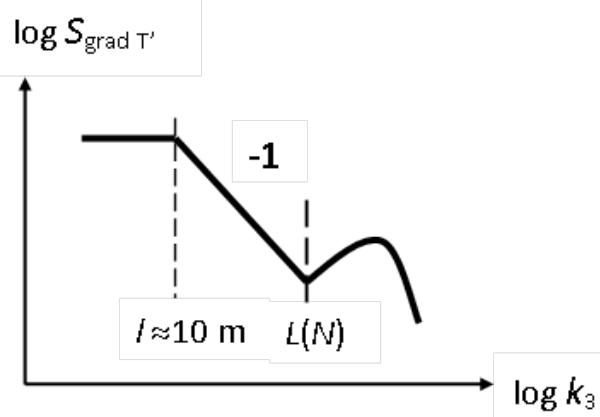

Fig. 1. Schematic form of the temperature gradient fluctuation spectrum vs a vertical wave number according to Gregg [4]. The scale $L$, depending on the Brent-Väisälä cyclical frequency $N_{c}$, corresponds to the effective scale of the turbulent patches

Many studies have been devoted to the research of the internal wave influence on turbulent exchange. The fundamental works of S. Thorpe [5, 6], T. Osborn [7] and others made a significant contribution to development of the concept about dynamic processes in stratified layers. From the recent studies, among many others an experimental study of internal waves in a thermocline [8] can be singled out. There the influence of the Kelvin waves on the turbulence intensity was discovered. A direct numerical modeling of the stably-stratified flow behavior, taking into account the fluid viscosity dependence on the temperature for various Reynolds numbers, is carried out in [9]. In [10] the spatial distribution of the turbulent energy dissipation rate and diapicnic mixing were studied based on observations in the Antarctic Circumpolar Current. It was shown that the dissipation rate is greater in the regions with a higher intensity of internal waves and also near-bottom irregularities.

In the present work a mathematical modeling of the turbulent patch effect on the form of the spectrum of pulsating values in a stably-stratified fluid layer with certain set of internal waves of different amplitudes and a random phase is carried out. This modeling was aimed to develop a method for analyzing experimental data, including the estimation of the effect of the number and size of patches formed inside the layer on the spectrum shape. The reliability check of the method described in [11] for determining the patch sizes from the local minimum of the vertical spectrum of the pulsation gradients was also performed. The important issue in the experimental spectra interpretation which the model study can also assist at, is the quantitative relationship relation between the intensity of turbulent pulsations and internal waves. The isolated elements of the results presented here were discussed in the thesis of one of the authors [12]. 


\section{Modeling of the turbulent patches}

As already noted, the internal wave breaking and the interlayer formation with increased turbulence intensity is one of the most important vertical exchange mechanisms in stratified layers. Since, as a rule, there are simultaneously many systems of internal waves in such layers, the localization of the turbulent patch formation is a random process. The main factor that increases break probability is the increasing of wave energy density per volume unit. Consequently, the number and extent of turbulent patches are determined by the internal wave intensity and in the final analysis characterize the vertical mixing efficiency. Real profiles of hydrophysical features reflect the presence of such intermixed regions formed as a result of the breaks which can clearly seen in Fig. 2.

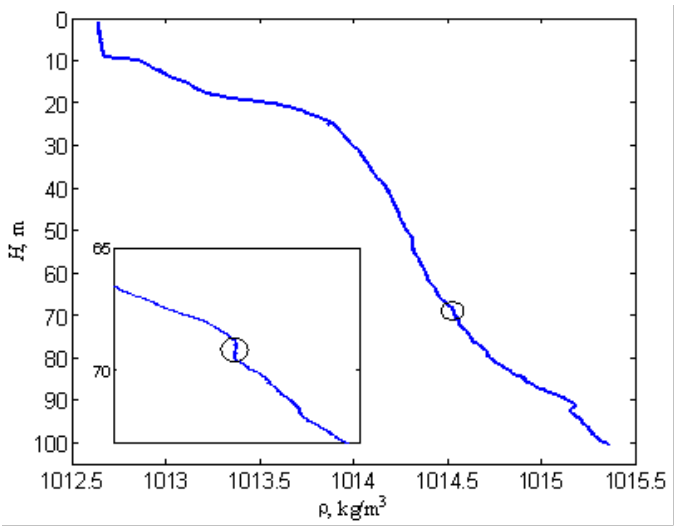

Fig. 2. Manifestations of turbulent patches on density profiles (according to the data of the Sigma-1 complex probing in the expedition on R/V Experiment to the northwestern part of the Black Sea in 2007) $[13,14]$. The circle shows the location of one patch, the inset presents an enlarged view

Fig. 3 shows the experimental energy spectra of temperature pulsations and temperature gradient pulsations. In the present case the temperature makes a decisive contribution to the density changing. As was shown in [4] the local minimum in the gradient spectrum corresponds to the effective scale of turbulent patches.
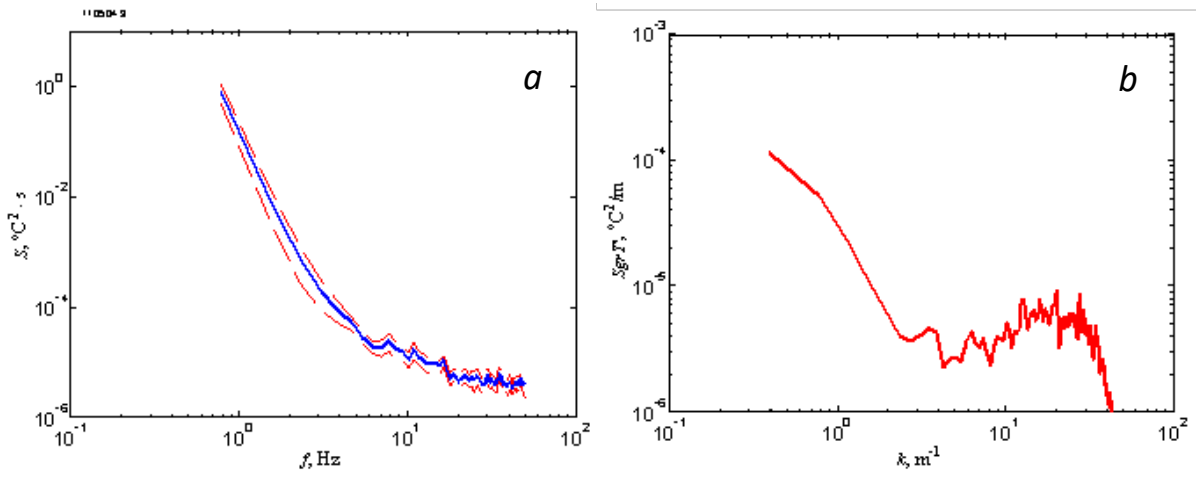

Fig. 3. Experimental spectra according to the Sigma-1 measurements in the Black Sea, R/V Akademik in 2004, station 18 [14]: (a) spectrum of temperature pulsations in the depth range of 41 $-83 \mathrm{~m}$, thin lines specify the $95 \%$ confidential interval, $(b)$ spectrum of temperature gradient pulsations vs vertical wave number 
To construct the mathematical model it should be mentioned that in the turbulent patch $\Delta=[\tau-L / 2, \tau+L / 2]$ of scale $L$ with a center in point $z=\tau$ the density can be expressed in the following way:

$$
\rho(z)=L \psi\left(\frac{z-\tau+L / 2}{L}\right)+c, \quad z \in \Delta,
$$

where $c$ is the constant defined by $\rho(z)$ values within its boundaries; $\psi$ is some universal function with the compact carrier concentrated in the interval $[0,1]$.

It is to be recalled that in order to validate the formula (1) in addition to Kolmogorov's hypotheses on the locally isotropic turbulence properties [15] the following argument was used.

Suppose $g(x)$ is some function, and $G(x)=g\left(L^{-1} x\right)$. The Fourier transformation is denoted by the symbol above:

$$
\hat{g}(k)=\int_{-\infty}^{\infty} g(x) \exp (-i k x) d x,
$$

where $k$ is the wave number. The following property of this transformation is known:

$$
\hat{G}(k)=L \hat{g}(L k) .
$$

It follows that the formula (1) is in complete agreement with the approximation obtained on the basis of the experimental data of the temperature gradient pulsations spectrum in the patch. This spectrum has the following form [14]:

$$
F(k)=\left|\frac{\partial \hat{T}^{\prime}}{\partial z}(k)\right|^{2}=\left\{\begin{array}{rr}
0, & 0<k \leq(2 \pi) / L \\
\gamma(L) k^{1 / 3}, & k>(2 \pi) / L
\end{array} .\right.
$$

The multiplier $\gamma(L)=L$ before $\psi$ in the formula (1) is found from the equation for the local minimum point $k_{\min }$ of the gradient spectrum in a layer with one turbulent patch of the scale $L$ :

$$
k^{2} \gamma(L) L \Phi(L k)=1,
$$

where the function $\Phi(x)=c_{w}^{-1}\left(\hat{\psi}(x)+x \hat{\psi}^{\prime}(x)\right) ; \quad c_{w}$ is some positive constant characterizing the wave processes occurring in the studied layer, while the experimental fact that $k_{\min }$ in case of one patch has the order $L^{-1}$ in relation to its scale, i.e. $k_{\min } \sim L^{-1}$ [14], is taken into account.

In accordance to the concept in [16, pp. 141-144], the function $\psi(z)$ describing the process in the "single" turbulent patch $[0,1]$ represents a certain sum of chirps, i.e. the functions of the following form:

$$
f_{\alpha, \beta, \zeta}(z)=A|z-\zeta|^{\alpha} \cos \left(B|z-\zeta|^{-\beta}\right), \alpha, \beta>0, \zeta \in(0,1),
$$

localized in the neighborhood of their centers $\zeta$ by multiplying by some finite nonnegative even function $\varphi(z-\zeta)$ satisfying the condition $\varphi(0)=1$. In the existing terminology $\alpha$ is called the Hölder exponent and $\beta$ is the oscillation exponent [16]. 
In [17] the following approximated formula was obtained:

$$
\hat{f}_{\alpha, \beta, \zeta}(v) \approx C(\alpha, \beta, A, B) v^{-\frac{2 \alpha+\beta+2}{2(\beta+1)}} e^{i \eta(v ; \alpha, \beta, A, B)},
$$

describing the chirp Fourier transformation behavior with parameters $\alpha, \beta$ in the region of central frequencies, where $C(\alpha, \beta, A, B)$ and $\eta(v, \alpha, \beta, A, B)$ are some known constant and function of the variable $v$, respectively. The formula (2) implies the spectrum decrease rate estimation:

$$
\left|\hat{f}_{\alpha, \beta, z_{0}}(v)\right|^{2} \sim v^{-\frac{2 \alpha+\beta+2}{\beta+1}} .
$$

On the basis of the estimation procedure of the Fourier transformation orders developed in [18], it can be concluded that the localization of the chirp by multiplying it by some infinitely differentiable function $\varphi(z-\zeta)$ with a carrier concentrated in some neighborhood of the center has no effect on the feasibility of relations (4) and (5).

Combining the expression (5) with the spectrum decreasing index - 5/3 [15] which follow from Kolmogorov's hypotheses, it can be concluded that in the turbulent zone the local amplitude $|z-\zeta|^{\alpha}$ and the local frequency $|z-\zeta|^{-\beta-1}$ are not arbitrary, but are related by a rigid analytical relation:

$$
\frac{2 \alpha+\beta+2}{\beta+1}=\frac{5}{3},
$$

from whence it follows:

$$
\beta=3 \alpha+\frac{1}{2} .
$$

Thus, this observation means that in the sum expressing the function $\psi(\mathrm{z})$, there are only those chirps in which the Hölder exponent and the oscillation exponent are binded by the relation (6), and the scheme itself is one-dimensional rather than two-dimensional, as was originally assumed in the paper [16].

In the discrete model $\alpha$ axis is divided by points $\alpha_{j}=\delta j, j=1,2 \ldots \mathrm{N}$. The corresponding set $\Omega_{j}$ of points $\zeta$ in which the chirp with parameters $\alpha_{j}$ and $\beta_{j}=3 \alpha_{j}$ $++1 / 2$ is present can be determined on the basis of some experimental data. In this case, the recalculations of data from the patch of certain scale $L$ to 1 are carried out according to the formula (1).

Complementing the hypotheses of local isotropic turbulence by assuming certain uniformity in the distribution of turbulent characteristics in a patch, it is obtained that in the discrete variant each set $\Omega_{j}$ is represented by some finite set $\left\{\zeta_{j}^{k_{j}}\right\}_{k_{j}=1}^{P_{j}}$ of the points uniformly distributed on the interval $[0,1]$. The number of such points $P_{j}$ for the patch $j$ is found from the condition of the model correspondence to the experimental data.

Considering that, based on the aforementioned assumption, the parameters $A$ and $B$ in the formula (3) should be statistically the same for all the chirps with centers at the points of one block $\Omega_{j}$, the following formula for the model description of the function $\psi(z)$ is obtained:

$$
\psi(z)=\sum_{j=1}^{N} \sum_{k_{j}=1}^{P_{j}} A_{j}\left|z-\zeta_{j}^{k_{j}}\right|^{\alpha_{j}} \cos \left(B_{j}\left|z-\zeta_{j}^{k_{j}}\right|^{-\left(3 a_{j}+1 / 2\right)}\right) \phi\left(z-\zeta_{j}^{k_{j}}\right) .
$$


In the initial model it is assumed that the parameters $A_{j}$ и $B_{j}$ are selected almost arbitrarily, but in such a way that the natural condition is satisfied from the physical viewpoint: the continuous norm of the first summand in the formula (1) should be incommensurably smaller than the addend, i. e. the density fluctuations should be much smaller than the mean value. In fact, taking into account the data on $C$ and $\eta$ from the formula (4), these parameters can be described by means of some single universal constant $c_{\text {turb }}$ which is responsible for the turbulence level in the considered patch or layer.

Another possible refinement of the model involves the replacement of the cosine function in the formula (1) by some linear combination of cosine and sine, i.e. with the introduction of the phase, leading to the following formula, taking into account the presence of internal waves and turbulent patches in the stratified layer:

$$
\psi_{\alpha, \beta}(z)=a z+\sum_{i=1}^{M} b_{i}^{\alpha} \sin \left(l_{i} z\right)+\theta(z)+\left[\sum_{j=1}^{N} \sum_{k=1}^{P} c_{j, k}^{\beta} \cos \left(\lambda_{j, k} z_{1 k}\right)+\xi_{k}\left(z_{1 k}\right)\right],
$$

where $a, b$ and $c$ are some dimensional coefficients; $\alpha$ and $\beta$ are the indices determining the spectrum change law of the corresponding process; $l$ and $\lambda$ are the wave numbers of internal waves and turbulent pulsations, respectively; $M$ and $P$ is the number of their harmonics; $N$ is the quantity of turbulent patches; $\theta$ and $\xi_{k}$ is white noise of different intensity; $z$ is the vertical coordinate; $z_{1}$ is the coordinate in the patch. The first term on the right side of the model series was added for the general correspondence to the required function to the real value (the presence of stratification) and was not taken into account when the spectrum calculating. The second and third terms correspond to the wave spectrum range, the fourth and fifth (in square brackets) ones correspond to the turbulent range.

\section{Results of the calculations}

To obtain a satisfactory agreement with experimental spectra in the given examples (Fig.3), up to 80 harmonics for internal waves and up to 50 for turbulent pulsations were used in the calculations using the formula (7). The model spectrum of internal waves with turbulence and in its absence is shown in Fig. 4. The scale $L$ of the patches in this case was set within the range from 0.1 to $L_{\max }=1.5 \mathrm{~m}$, the locations were at regular intervals throughout the layer. In modeling of the turbulent patches, the function with a compact carrier limiting the range of pulsations to these patch boundaries was used.

One of the problems solved by such modeling was the validation of the method described in [11] used in calculating the mean size of turbulent patches $<L>$ for the vertical temperature spectra and its gradient. Fig. 5 shows examples of the spectra of gradients with different specified mean sizes of model patches. A good correspondence with the scales $L_{k}$, determined by the local minimum on the spectrum can be seen. Consequently, the validity of the method used to estimate $<L>$ is confirmed by modeling.

Estimation of the spectrum form variation depending on the number of patches, their distribution in size, depth localization, etc. by means of modeling is also very useful. Fig. 6 shows a number of model spectra calculated for different scales of turbulent patches, as well as for different intensities of both internal waves and turbulence in the patches. 


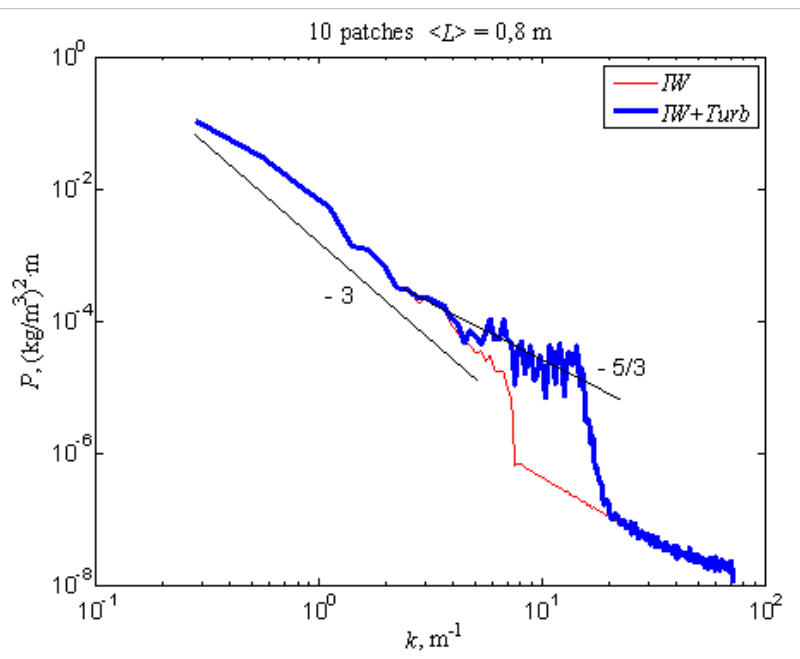

Fig. 4. Model energy spectra of internal waves $(I W)$ and the total signal of internal waves and turbulence, concentrated within the patches $(I W+T u r b)$. The number of patches was 10 , they were distributed evenly throughout the entire layer and the mean scale of the patches was $\langle L>\approx 0.8 \mathrm{~m}$

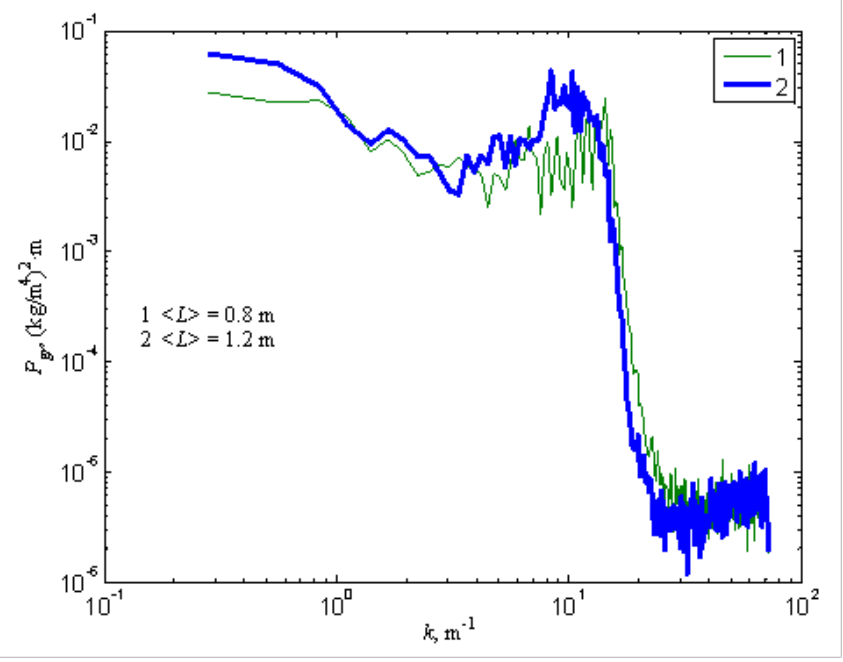

Fig. 5. Model spectra of the pulsation gradients for various mean scales of turbulent patches. The number of patches in both cases was 10. $<L>$ is the specified mean scale of the patches

Calculation of model spectra in different combinations of input parameters permitted to determine certain features of the effect of the specified characteristics of patches and internal waves on the spectra form:

- Size of the patches influences on the position of the local minimum on the spectrum: mean size increase whole leads in the large to minimum shift to the longwave spectrum range but in some cases there may be deviations from this rule which is determined by the turbulence intensity inside the patches.

- Arrangement of patches by size (increase or decrease with depth) does not affect the spectrum form. 
- An increase in the quantity of patches on retention of the mean size leads to an increase in the spectral level in the range of turbulent patches scales that may influence on the position of the minimum.

- The position of the local minimum depends on the ratio of the oscillations intensity due to the internal waves and turbulence; at the same time the total spectrum level also changes.
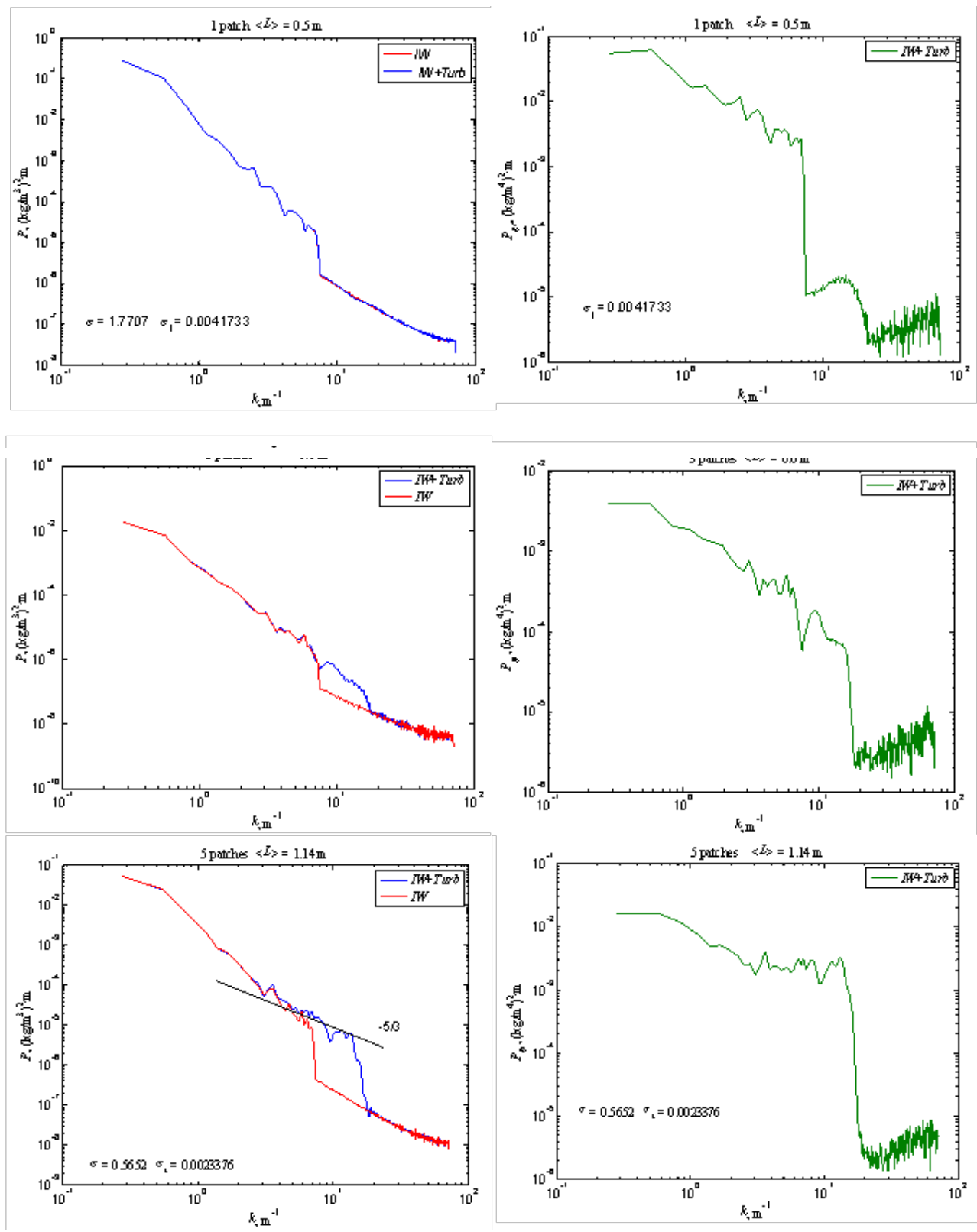

Fig. 6. Model pulsation spectra (left) and pulsation gradient spectra (right) for a different number of turbulent patches and various energy levels, $\sigma$ is the total signal dispersion, $\sigma t$ is the dispersion of turbulent pulsations 

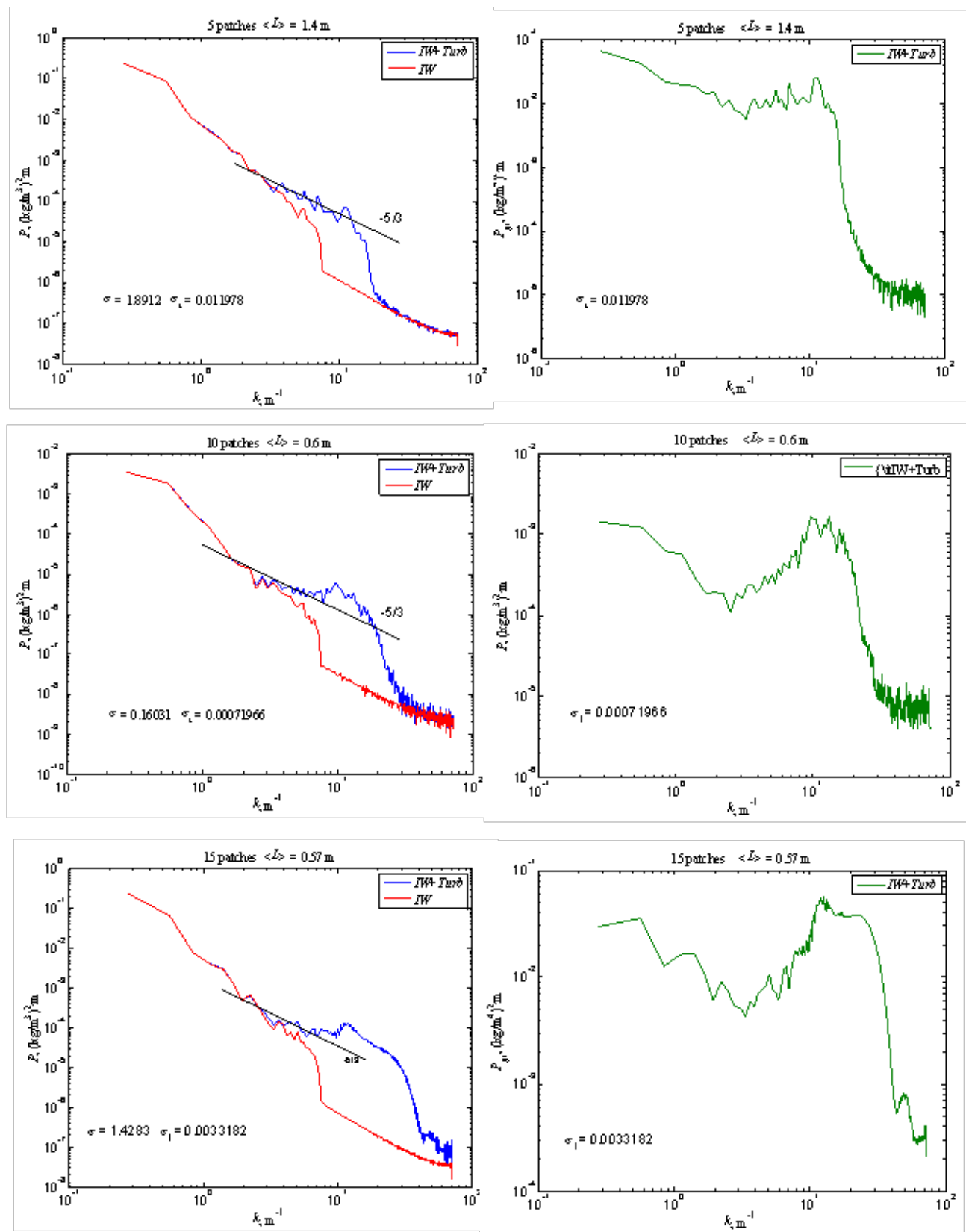

Fig. 6. (continuation)

It should be highlighted the noted features are valid on average. The individual realizations showed a moderate scatter both in the internal wave energy levels and in the form and level of the turbulent pulsation spectrum, since each separate calculation depends on a random set of oscillations.

Consequently, the used approach to microstructural spectra model construction allows obtaining the results close to real data and, by comparison, making estimations of the turbulent characteristics in the in situ data. This model relevance 
lies both in the verification of theoretical conclusions and in the discovery of new regularities in the turbulent patch formation and dynamics in stratified layers. A comparative analysis of the experimental and model spectra makes it possible to estimate the quantity of turbulent patches and the mean intensity of turbulent pulsations in the studied layer.

Acknowledgements. The work was fulfilled within the framework of the State Order No. 0827-2014-0010 under the partial support of the RFBR Grant No. 1605-00664.

\section{REFERENCES}

1. Kamenkovich, V.M. and Monin, A.S. eds., 1978. Fizika Okeana, V.2. Gidrodinamika Okeana [Ocean Physics, V. 2. Ocean Hydrodynamics]. Moskow: Nauka, 455 p. (in Russian).

2. Wunsch, C. and Ferrari, R., 2004. Vertical Mixing, Energy and the General Circulation of the Ocean. Annu. Rev. Fluid Mech., [e-journal] 36(1), pp. 281-314. doi:10.1146/annurev.fluid.36.050802.122121

3. Hebert, D., Moum, J.N., Paulson, C.A. and Caldwell, D.R., 1992. Turbulence and Internal Waves at the Equator. Part II: Details of a Single Event. J. Phys. Oceanogr., [e-journal] 22(11), pp. 1346-1356. doi:10.1175/1520-0485(1992)022<1346:TAIWAT>2.0.CO;2

4. $\quad$ Gregg, M.C., 1989. Scaling Turbulent Dissipation in the Thermocline. J. Geophys. Res., [e-journal] 94(C7), pp. 9686-9698. doi:10.1029/JC094iC07p09686

5. Thorpe, S.A., 1973. Experiments on Instability and Turbulence in a Stratified Shear Flow. J. of Fluid Mechanics, [e-journal] 61(4), pp. 731-751. doi:10.1017/S0022112073000911

6. Thorpe, S.A., 1987. Transitional Phenomena and the Development of Turbulence in Stratified Fluids: A Review. J. Geophys. Res., [e-journal] 92(C5), pp. 5231-5248. doi:10.1029/JC092iC05p05231.

7. Osborn, T.R., 1974. Vertical Profiling of Velocity Microstructure. J. Phys. Oceanogr., [e-journal] 4(1), pp. 109-115. doi:10.1175/1520-0485(1974)004<0109:VPOVM>2.0.CO;2

8. Preusse, M., Peeters, F. and Lorke, A., 2010. Internal Waves and the Generation of Turbulence in the Thermocline of a Large Lake. Limnol. Oceanogr., 55(6), pp. 2353-2365.

9. Zonta, F., Onorato, M. and Soldati, A., 2012. Turbulence and Internal Waves in StablyStra-tified Channel Flow with Temperature-Dependent Fluid Properties. J. Fluid Mech., [e-journal] (697), pp. 175-203. doi:10.1017/jfm.2012.51

10. Waterman, S., Naveira-Carabato, A.C. and Polzin, K.L., 2013. Internal Waves and Turbulence in the Antarctic Circumpolar Current. J. Phys. Oceanogr., [e-journal] 43(2), pp. 259-282. doi:10.1175/JPO-D-11-0194.1

11. Samodurov, A.S., Lubitsky, A.A. and Panteleev, N.A., 1995. Contribution of Breaking Internal Waves to Structure Formation, Energy Dissipation, and Vertical Diffusion in the Ocean. Physical Oceanography, [e-journal] 6(3), pp. 177-190. doi:10.1007/BF02197516

12. Chukharev, A.M., 2014. Vklad Osnovnykh Mekhanizmov Generatsiy Turbulentnosti $v$ Vertikalnyy Obmen $v$ Deyatelnom Sloe Morya [Contribution of the Basic Mechanisms of Turbulence Generation to Vertical Exchange in the Active Sea Layer]. Doctorate Thesis. Sevastopol, $275 \mathrm{p}$.

13. Samodurov, A.S., Dykman, V.Z., Barabash, V.A., Efremov, O.I., Zubov, A.G., Pavlenko, O.I., Chukharev, A.M., 2005. "Sigma-1" Measuring Complex for the Investigation of SmallScale Characteristics of Hydrophysical Fields in the Upper Layer of the Sea. Physical Oceanography, [e-journal] 15(5), pp. 311-322. https://doi:org/10.1007/s11110-006-0005-1

14. Samodurov, A.S. and Chukharev, A.M., 2008. Experimental Estimation of the Coefficient of Vertical Turbulent Exchange in a Stratified Layer of the Black Sea near the Continental Slope. Physical Oceanography, [e-journal] 18(6), pp. 308-318. doi:10.1007/s11110-0099032-z 
15. Monin, A.S. and Yaglom, A.M., 2007. Statistical Fluid Mechanics, Volume II: Mechanics of Turbulence. Mineola, NY: Dover Publications, 896 p.

16. Jaffard, S., Meyer, Y. and Ryan, R.D., 2001. Wavelets. Tools for Science \& Technology. Philadelphia: SIAM, $255 \mathrm{p}$.

17. Innocent, J.-M. and Torrésani, B., 1996. A Multiresolution Strategy for Detecting Gravitational Waves Generated by Binary Coalescence. Internal Report CPT-96/P.3379, CPT-CNRS. Marseille, 13 p.

18. Runovski, K. and Schmeisser, H.-J., 2015. Moduli of Smoothness Related to the LaplaceOperator. J. Fourier Analys. Applicat., [e-journal] 21(3), pp. 449-471. doi:10.1007/s00041014-9373-y 